\title{
ON THE CONSTRUCTION OF THE FORWARD AND BACKWARD SOLUTION SPACE OF A DISCRETE TIME AR-REPRESENTATION
}

\author{
N. P. Karampetakis * \\ * Department of Mathematics, Aristotle University of \\ Thessaloniki, Thessaloniki 54006, Greece, karampet@auth.gr
}

\begin{abstract}
The main purpose of this work is to construct the forward and backward solution space of a nonregular discrete time AR-representation i.e. $A(\sigma) \xi(k)=0$, in a closed interval $[0, N]$ where $A(\sigma)$ is a polynomial matrix and $\sigma$ is the forward shift operator. The construction of the behavior is based on the structural invariants of the polynomial matrix which describe the AR-representation, i.e. the finite and infinite elementary divisors and the right minimal indices of $A(\sigma)$.
\end{abstract}

Keywords: Autotegressive models, behaviour, boundary conditions, discrete time systems, indices

Consider a system of linear homogeneous difference and algebraic equations described in matrix form by :

$$
A(\sigma) \xi_{k}=0
$$

where $\sigma$ denotes the forward shift operator i.e. $\sigma \xi_{k}=\xi_{k+1}, A(\sigma)=A_{0}+A_{1} \sigma+\cdots+A_{q} \sigma^{q} \in$ $R[\sigma]^{p \times m}$ with $\operatorname{rank}_{R(\sigma)} A(\sigma)=r \leq \min (p, m)$ and $\xi_{k}:[0, N] \rightarrow R^{m}$. We call the set of equations (1) an AR-Representation of $B_{D}$ (behavior) where $B_{D}$ is defined as :

$$
B_{D}:=\left\{\begin{array}{c}
\xi_{k}:[0, N] \rightarrow R^{m} \\
(1) \text { is satisfied } \forall k \in[0, N]
\end{array}\right\}
$$

In case where $A(\sigma)$ is regular the solution vector space of (1) consists of $r q$ i.e. the total number of finite and infinite elementary divisors (order accounted for), linearly independent forward and backward solutions (Antoniou et al., 1998). In case now where $A(\sigma)$ is nonregular, the space $B_{D}$ contains a number of linear independent forward and backward solutions that depends on $\mathrm{N}$ and are due to the right null space of $A(\sigma)$ (Karampetakis, 2001). If we now correspond all the forward and backward solutions which are due to a specific boundary value (initial-final condition) to an element $\left[\xi_{k}\right]$ then the behaviour space $B_{D}$ is divided into equivalence classes and a new space is created, named $\hat{B}_{D}$, whose dimension is proved to be the total number of the finite elementary divisors $(n)$, infinite elementary divisors $(\mu)$, plus two times the right minimal indices $(\epsilon)$ of $A(\sigma)$ (order accounted for) i.e. $n+\mu+2 \epsilon$ (Karampetakis, 2001). In this paper we give a specific construction of the solution cpace $\hat{B}_{D}$ by finding $n+\epsilon$ linearly independent forward solutions representatives due to the finite elementary divisors and the right minimal indices of $A(\sigma)$ and $\mu+\epsilon$ linearly independent backward solution representatives due to the infinite elementary divisors and the right minimal indices of $A(\sigma)$. Finally, the construction of the basis that produces the space $\hat{B}_{D}$ helps us to construct additionally the space $B_{D}$.

\section{PRELIMINARY RESULTS}

Applying the $\mathrm{Z}$ transform of $\xi(k)$ given by (Freeman, 1965) i.e. $\xi(z) \stackrel{\text { def }}{=} Z[\xi(k)]=\sum_{k=0}^{N} \xi(k) z^{-k}$ to $(1)$ we get : 


$$
\begin{aligned}
& A(z) \xi(z)=\left[\begin{array}{llll}
z^{q} I_{p} & \cdots & z I_{p} & I_{p}
\end{array}\right] \times \\
& \times\left[\begin{array}{cccc}
A_{q} & 0 & \cdots & 0 \\
A_{q-1} & A_{q} & \cdots & 0 \\
\vdots & \vdots & \ddots & \vdots \\
A_{1} & A_{2} & \cdots & A_{q}
\end{array}\right]\left[\begin{array}{c}
\xi(0) \\
\xi(1) \\
\vdots \\
\xi(q-1)
\end{array}\right]+ \\
& +\left[\begin{array}{llll}
z^{-N} I_{p} & \cdots & z^{-N+q-2} I_{p} & z^{-N+q-1} I_{p}
\end{array}\right] \times \\
& \times\left[\begin{array}{cccc}
A_{0} & 0 & \cdots & 0 \\
A_{1} & A_{0} & \cdots & 0 \\
\vdots & \vdots & \ddots & \vdots \\
A_{q-1} & A_{q-2} & \cdots & A_{0}
\end{array}\right]\left[\begin{array}{c}
\xi(N) \\
\xi(N-1) \\
\vdots \\
\xi(N-q+1)
\end{array}\right] \\
& =:\left(z^{q} I_{p}\right) \Re_{q} \tilde{\xi}(0)+\left(z^{-N} I_{p}\right) \Re_{0} \tilde{\xi}(N)
\end{aligned}
$$

Let also a right minimal polynomial basis ${ }^{1}$ consisted by the following polynomial vectors

$$
\begin{gathered}
\tilde{u}_{i}(\sigma):=\tilde{u}_{i, 0}+\tilde{u}_{i, 1} \sigma+\cdots+\tilde{u}_{i, \varepsilon_{i}} \sigma^{\varepsilon_{i}} \\
i=r+1, r+2, \ldots, m
\end{gathered}
$$

Then the rational vectors of the form

$$
\begin{gathered}
\hat{u}_{i}(\sigma):=\tilde{u}_{i, 0} \frac{1}{\sigma^{\varepsilon_{i}}}+\tilde{u}_{i, 1} \frac{1}{\sigma^{\varepsilon_{i}-1}}+\cdots+\tilde{u}_{i, \varepsilon_{i}} \\
i=r+1, r+2, \ldots, m
\end{gathered}
$$

still constitutes a right minimal proper basis of $A(\sigma)$. Let now

$$
X=\left\{\begin{array}{c}
\xi(k): \tilde{\xi}(0) \in \operatorname{Ker}\left[\Re_{q}\right] \\
\text { and } \tilde{\xi}(N) \in \operatorname{Ker}\left[\Re_{0}\right] \\
\xi(k)=\sum_{i=0}^{k} \hat{u}_{r+1}(i) z_{1}(k-i)+ \\
+\cdots+\sum_{i=0}^{k} \hat{u}_{m}(i) z_{m-r}(k-i)
\end{array}\right\}
$$

where $z_{i}(k), i=1,2, \ldots, m-r$ are arbitrary discrete time functions and $\hat{u}_{i}(k)=Z^{-1}\left[\hat{u}_{i}(z)\right], i=$ $r+1, \ldots, m$, be the solution space of (1) (Karampetakis, 2001) which comes under the above specific initialfinal conditions.

Define now the following relation $R$ between the solutions of $B_{D}$

$$
\begin{gathered}
R\left(\xi_{1}(k), \xi_{2}(k)\right):= \\
=\left\{\begin{array}{c}
\left(\xi_{1}(k), \xi_{2}(k)\right): \xi_{1}(k)-\xi_{2}(k) \in X \\
\text { where } \xi_{1}(k), \xi_{2}(k) \in B_{D}
\end{array}\right\}
\end{gathered}
$$

Then the relation (4) defines an equivalence relation (Karampetakis, 2001). We call an equivalence class of the element $\xi(k) \in B_{D}$, and we denote

\footnotetext{
1 For the simplicity of the proofs of the main Theorems we give a specific construction of the minimal polynomial basis in (7), although in practice other constructions may also be used i.e. (Beelen, 1987).
}

this with $[\xi(k)]_{R}$, the set of all the elements of $B_{D}$ which are equivalent to $\xi(k)$ or equivalently

$$
[\xi(k)]_{R}:=\xi(k) \oplus X
$$

We can see that any equivalence class of an element $\xi(k)$ gives all the solutions of (1) under some specific initial-final conditions. In the case where $A(\sigma)$ has no right kernel then every equivalence class is composed either by a unique element or no element (due to left null space) contrary to the nonregular case where to each equivalence class corresponds an arbitrary number of elements of $B_{D}$. We conclude therefore, that $B_{D}$ is divided into equivalence classes which are defined by (5). Define now the following sum and product between equivalence classes of the form (5) :

$$
\begin{aligned}
{\left[\xi_{1}(k)\right]_{R}+\left[\xi_{2}(k)\right]_{R} } & :=\left[\xi_{1}(k)+\xi_{2}(k)\right]_{R} \\
\lambda[\xi(k)]_{R} & =[\lambda \xi(k)]_{R} \quad \lambda \in R
\end{aligned}
$$

Theorem 1. (Karampetakis, 2001) The space which is spanned by the equivalence classes defined in (5) is a vector space $\hat{B}_{D}:=B_{D} / R$ and has dimension

$$
f:=\operatorname{dim} \hat{B}_{D}=n+\mu+2 \varepsilon
$$

In the following section we shall try to determine a basis for the vector space $\hat{B}_{D}$ in terms of the structural invariants of the polynomial matrix $A(s)$ and thus to find out the solution space $B_{D}$.

\section{CONSTRUCTION OF THE BEHAVIOR OF A DISCRETE TIME AR-REPRESENTATION}

In this section we shall try to define $n$ linearly independent forward solutions of (1) due to the , finite elementary divisors of $A(s), \mu$ linearly independent backward solutions of (1) due to the infinite elementary divisors of $A(s), \epsilon$ linearly independent forward solutions of (1) due to the right minimal indices of $A(s)$ and $\epsilon$ linearly independent backward solutions of (1) due to right minimal indices of $A(s)$.

\subsection{Finite elementary divisors and solutions of discrete time AR-representations}

Let us assume that $A(\sigma)$ has $k$ distinct eigenvalues $\lambda_{1}, \lambda_{2}, \ldots, \lambda_{k}$ where for simplicity of notation we assume that $\lambda_{i} \in R, i \in k$ and let

$$
\begin{gathered}
U_{L}(\sigma) A(\sigma) U_{R}(\sigma)= \\
=\operatorname{blockdiag}\left[I_{z-1}, f_{z}(\sigma), f_{z+1}(\sigma), \ldots, f_{r}(\sigma), 0_{p-r, m-r}\right]
\end{gathered}
$$


$1 \leq z \leq r$ be the Smith form of $A(\sigma)$ (in $C$ ) where $f_{i}(\sigma) \in R[\sigma]$ are the invariant polynomials of $A(\sigma)$ and $f_{i}(\sigma) / f_{i+1}(\sigma) i=z, z+1, \ldots, r-$ 1. Assume that the partial multiplicities of the eigenvalues $\lambda_{i} \in R, i \in k$ are $0 \leq \sigma_{i, z} \leq$ $\sigma_{i, z+1} \leq \cdots \leq \sigma_{i, r}$. Let $u_{j}(\sigma) \in R[\sigma]^{m \times 1}, j \in$ $R$ be the columns of $U_{R}(\sigma)$ and $u_{j}^{(q)}(\sigma):=$ $\left(d^{q} / d s^{q}\right) u_{j}(\sigma), q=0,1, \ldots, \sigma_{i, j}-1$. Let also

$$
x_{j, q}^{i}:=\frac{1}{q !} u_{j}^{(q)}\left(\lambda_{i}\right) \quad i \in k \text { and } j=z, z+1, . ., r
$$

Define the vector valued functions

if $\lambda_{i} \neq 0$

$$
\begin{gathered}
\xi_{j, q}^{i}(k):=\lambda_{i}^{k} x_{j, q}^{i}+\cdots+\left(\begin{array}{c}
k \\
q
\end{array}\right) \lambda_{i}^{k-q} x_{j, 0}^{i} \\
i \in k ; j=z, z+1, \ldots, r \quad ; q=0,1, \ldots, \sigma_{i, j}-1
\end{gathered}
$$

if $\lambda_{i}=0$

$$
\begin{gathered}
\xi_{j, q}^{i}(k):=\Delta(k) x_{j, q}^{i}+\cdots+\Delta(k-q) x_{j, 0}^{i} \\
i \in k ; j=z, z+1, \ldots, r \quad ; q=0,1, \ldots, \sigma_{i, j}-1
\end{gathered}
$$

Let

$$
\begin{aligned}
\Psi_{i, j}(k):= & {\left[\xi_{j, 0}^{i}(k) \xi_{j, 1}^{i}(k) \cdots \xi_{j, \sigma_{i, j}-1}^{i}(k)\right] } \\
C_{i, j}:= & {\left[\begin{array}{ccccc}
x_{j, 0}^{i} & x_{j, 1}^{i} & \cdots & x_{j, \sigma_{i, j}-2}^{i} & x_{j, \sigma_{i, j}-1}^{i}
\end{array}\right] } \\
J_{i, j}:= & {\left[\begin{array}{ccccc}
\lambda_{i} & 1 & \cdots & 0 & 0 \\
0 & \lambda_{i} & \cdots & 0 & 0 \\
\vdots & \vdots & \ddots & \vdots & \vdots \\
0 & 0 & \cdots & \lambda_{i} & 1 \\
0 & 0 & \cdots & 0 & \lambda_{i}
\end{array}\right] \in R^{\sigma_{i, j} \times \sigma_{i, j}} } \\
& i \in k ; j=z, z+1, \ldots, r
\end{aligned}
$$

and

$$
\begin{gathered}
\Psi_{i}^{F}(k):=\left[\begin{array}{llll}
\Psi_{i, z}(k) & \Psi_{i, z+1}(k) & \cdots & \Psi_{i, r}(k)
\end{array}\right] \\
C_{i}^{F}:=\left[\begin{array}{lllll}
C_{i, z}(k) & C_{i, z+1}(k) & \cdots & C_{i, r}(k)
\end{array}\right] \\
J_{i}^{F}:=\text { blockdiag }\left[\begin{array}{lllll}
J_{i, z}(k) & J_{i, z+1}(k) & \cdots & J_{i, r}(k)
\end{array}\right]
\end{gathered}
$$

where $m_{i}:=\sigma_{i, z}+\sigma_{i, z+1}+\cdots \sigma_{i, r} i \in k$. Finally let

$$
\begin{gathered}
\Psi_{F}^{D}(k):=\left[\begin{array}{llll}
\Psi_{1}^{F}(k) & \Psi_{2}^{F}(k) & \cdots & \Psi_{k}^{F}(k)
\end{array}\right] \\
C_{F}^{D}:=\left[\begin{array}{lllll}
C_{1}^{F}(k) & C_{2}^{F}(k) & \cdots & C_{k}^{F}(k)
\end{array}\right] \\
J_{F}^{D}:=\text { blockdiag }\left[\begin{array}{lllll}
J_{1}^{F}(k) & J_{2}^{F}(k) & \cdots & J_{k}^{F}(k)
\end{array}\right]
\end{gathered}
$$

where $n:=\operatorname{deg}\left[\prod_{j=z}^{r} f_{j}(\sigma)\right]=\operatorname{deg}\left|S_{R(\sigma)}^{C}\right|$. Then we have the following
Theorem 2. The columns of the following matrix

$\Psi_{F}^{D}(k):=\left[\begin{array}{lllll}\Psi_{1}^{F}(k) & \Psi_{2}^{F}(k) & \cdots & \Psi_{k}^{F}(k)\end{array}\right]=C_{F}^{D}\left(J_{F}^{D}\right)^{k}$

constitute a solution subspace $B_{F}^{D}$ of $B_{D}-X$ i.e. $B_{F}^{D} \subseteq B_{D}-X$ with dimension

$\operatorname{dim} B_{F}^{D}=n:=\left\{\begin{array}{c}\text { total sum of the degrees of the } \\ \text { finite elementary divisors of } A(\sigma)\end{array}\right\}$

Proof. It is easily seen (see also (Gogberg et al., 1982)) that the pair $\left(C_{F}^{D}, J_{F}^{D}\right)$ constitute a finite spectral pair of $A(\sigma)$ which satisfy the following :

$\sum_{k=0}^{q} A_{k} C_{F}^{D}\left(J_{F}^{D}\right)^{k}=0 ; \operatorname{rank} \operatorname{col}\left(C_{F}^{D}\left(J_{F}^{D}\right)^{k}\right)_{k=0}^{n-1}=n$

and, therefore, the columns of the matrix $\Psi_{F}^{D}(k)$ satisfy the equation (1). In order to prove that $B_{F}^{D} \subseteq B_{D}-X$ we have to show that the columns of the matrix $\Psi_{F}^{D}(k)$ do not belong to $X$ or otherwise

$$
\begin{aligned}
& {\left[C_{F}^{T}\left(C_{F} J_{F}\right)^{T} \cdots\left(C_{F} J_{F}^{q-1}\right)^{T}\right]^{T} \notin \operatorname{Ker}\left[R_{q}\right]} \\
& {\left[\left(C_{F} J_{F}^{N}\right)^{T}\left(C_{F} J_{F}^{N}\right)^{N-1} \ldots\left(C_{F} J_{F}^{N}\right)^{N-q}\right] \notin \operatorname{Ker}\left[R_{0}\right]}
\end{aligned}
$$

The proof is not presented here in order to avoid including a lot of technicalities. However, it is based a) on the specific selection of the right minimal basis selected in the sequel and b) on the linearly independence of the columns of the transforming matrix $U_{R}(s)$ defined above. The proof follows similar lines to the ones for the continuous time case presented in (Karampetakis, 1993) (Chapter 6).

Any other finite spectral pair will also define an isomorphic space to $B_{F}^{D}$. However our intention is twofold : a) to give the reader a method for the construction of the finite spectral pair and b) to simplify some of the proofs with the specific form of this spectral pair.

\subsection{Infinite elementary divisors and solutions of}

discrete time AR-representations

Define the "dual" polynomial matrix $\tilde{A}(w)$ of $A(\sigma)$ as

$\tilde{A}(w):=A_{0} w^{q}+A_{1} w^{q-1}+\cdots+A_{q} \in R[w]^{p \times m}$

Let $\tilde{U}_{L}(w) \in R(w)^{p \times p}, \tilde{U}_{R}(w) \in R(w)^{m \times m}$ be rational matrices having no poles and zeros at $w=0$ and such that 


$$
\begin{gathered}
\tilde{U}_{L}(w) \tilde{A}(w) \tilde{U}_{R}(w)=S_{\tilde{A}(w)}^{0}(w)= \\
=\operatorname{blockdiag}\left[I_{d-1}, z^{\mu_{d}}, \ldots, z^{\mu_{r}}, 0_{p-r, m-r}\right]
\end{gathered}
$$

where $S_{\tilde{A}(w)}^{0}(w)$ is the Smith form of $\tilde{A}(w)$ at $w=$ 0 . Let now $\tilde{U}_{R}(w)=\left[\begin{array}{lllll}\tilde{u}_{1}(w) & \tilde{u}_{2}(w) & \cdots & \tilde{u}_{m}(w)\end{array}\right]$ where $\tilde{u}_{j}(w) \in R(w)^{m \times 1}$ and $\tilde{u}_{j}^{(i)}(w), \tilde{A}^{(i)}(w)$ be the qth derivatives of $\tilde{u}_{j}(w)$ and $\tilde{A}(w)$ with respect to $w$ for $i=0,1, \ldots, \mu_{j}-1$ and $j=d, d+$ $1, \ldots, r$ where $\mu_{j}$ are the multiplicities of the zeros of $\tilde{A}(w)$ at $w=0$ or equivalently the degrees of the infinite elementary divisors. Define

$$
x_{j, i}:=\frac{1}{i !} \tilde{u}_{j}^{(i)}(0)
$$

for $i:=0,1, \ldots, \mu_{j}-1$ and $j=d, d+1, \ldots, r$. Then for final conditions

$$
\left[\begin{array}{c}
\xi(N) \\
\xi(N-1) \\
\vdots \\
\xi(N-q+1)
\end{array}\right]=\left[\begin{array}{c}
x_{j, i} \\
\vdots \\
x_{j, 0} \\
0_{(q-i-1), 1}
\end{array}\right]
$$

we obtain respectively the linearly independent backward solutions

$$
\xi_{j, i}^{B}(k):=x_{j, i} \delta(N-k)+\cdots+x_{j, 0} \delta(N-(k-i))
$$

Define the vector valued functions

$$
\begin{aligned}
\xi_{j, i}^{B}(k): & =x_{j, i} \delta(N-k)+\cdots+x_{j, 0} \delta(N-(k-i)) \\
i: & =0,1, \ldots, \mu_{j}-1 \text { and } j=k, k+1, \ldots, r
\end{aligned}
$$

Let

$$
\begin{aligned}
\Psi_{j}^{B}(k): & =\left[\begin{array}{llll}
\xi_{j, 0}^{B}(k) & \xi_{j, 1}^{B}(k) & \cdots & \xi_{j, \mu_{j}-1}^{B}(k)
\end{array}\right] \\
C_{j}^{B}: & =\left[\begin{array}{llll}
x_{j, 0} & x_{j, 1} & \cdots & x_{j, \mu_{j}-1}
\end{array}\right] \\
J_{j}^{B}: & =\left[\begin{array}{ccccc}
0 & 1 & \cdots & 0 & 0 \\
0 & 0 & \cdots & 0 & 0 \\
\vdots & \vdots & \ddots & \vdots & \vdots \\
0 & 0 & \cdots & 0 & 1 \\
0 & 0 & \cdots & 0 & 0
\end{array}\right] \in R^{\mu_{j} \times \mu_{j}}
\end{aligned}
$$

where $j=d, d+1, \ldots, r$ and

$$
\begin{gathered}
\Psi_{B}^{D}(k):=\left[\begin{array}{llll}
\Psi_{k}^{B}(k) & \Psi_{k+1}^{B}(k) & \cdots & \Psi_{r}^{B}(k)
\end{array}\right] \\
C_{B}^{D}:=\left[\begin{array}{lllll}
C_{k}^{B}(k) & C_{k+1}^{B}(k) & \cdots & C_{r}^{B}(k)
\end{array}\right] \\
J_{B}^{D}:=\text { blockdiag }\left[\begin{array}{lllll}
J_{k}^{B}(k) & J_{k+1}^{B}(k) & \cdots & J_{r}^{B}(k)
\end{array}\right]
\end{gathered}
$$

where $\mu:=\sum_{j=d}^{r} \mu_{j}$. Then we have the following
Theorem 3. The columns of the following matrix

$\Psi_{B}^{D}(k):=\left[\Psi_{k}(k) \Psi_{k+1}(k) \cdots \Psi_{r}(k)\right]=C_{B}^{D}\left(J_{B}^{D}\right)^{N-k}$

constitute a solution subspace $B_{B}^{D}$ of $B_{D}-X$ i.e. $B_{B}^{D} \subseteq B_{D}-X$ with dimension

$\operatorname{dim} B_{B}^{D}=\mu:=\left\{\begin{array}{c}\text { total sum of the degrees of the } \\ \text { infinite elementary divisors }\end{array}\right\}$

Proof. It is easily seen that the pair $\left(C_{B}^{D}, J_{B}^{D}\right)$ constitutes an infinite spectral pair of $A(\sigma)$ which satisfies the following :

$\sum_{k=0}^{q} A_{k} C_{B}^{D}\left(J_{B}^{D}\right)^{N-k}=0 ; \operatorname{rank} \operatorname{col}\left(C_{B}^{D}\left(J_{B}^{D}\right)^{N-k}\right)_{k=0}^{\mu-1}=\mu$

and, therefore, the columns of the matrix $\Psi_{B}^{D}(k)$ satisfy the equation (1). Similar comments to the ones in the proof of Theorem 2, also apply here.

Any other infinite spectral pair which corresponds to infinite elementary divisors will also define an isomorphic space to $B_{B}^{D}$.

\subsection{Right minimal indices and solutions of discrete} time AR-representations

$A(\sigma) \in R[\sigma]^{p \times m}$ according to our assumption has rank $r \leq \min \{p, m\}$ and therefore the dimension of the right null space of $A(\sigma)$ is equal to $m-$ $r$. Consider a minimal polynomial bases ${ }^{2}$ of the right null space of $A(\sigma)$, let

$$
\left[\bar{u}_{r+1}(\sigma) \bar{u}_{r+2}(\sigma) \cdots \bar{u}_{m}(\sigma)\right]
$$

The greatest degrees of the columns $\bar{u}_{i}(\sigma), i=r+$ $1, r+2, \ldots, m$ let $\left\{\varepsilon_{r+1}, \varepsilon_{r+2}, \ldots, \varepsilon_{m}\right\}$ are called right minimal indices of $A(\sigma)$. Let also

$$
\begin{gathered}
x_{j, i}:=\frac{1}{i !} \bar{u}_{j}^{(i)}(0) \\
i=0,1, \ldots, \varepsilon_{i}-1 \text { and } j=r+1, r+2, \ldots, m
\end{gathered}
$$

Define the vector valued functions

$$
\begin{gathered}
\xi_{j, i}^{F}(k):=\delta(k) x_{j, i}+\cdots+\delta(k-i) x_{j, 0} \\
i=0,1, . ., \varepsilon_{j}-1 \text { and } j=r+1, r+2, \ldots, m
\end{gathered}
$$

Let

\footnotetext{
2 The last $m-r$ columns of the transforming matrix $U_{R}(\sigma)$ defined in subsection 2.1, constitute a basis of the right kernel of $A(\sigma)$. Under certain unimodular transformations i.e. column reduceness, we may do the above basis minimal.
} 


$$
\begin{aligned}
\Psi_{j}^{F}(k) & :=\left[\begin{array}{lllll}
\xi_{j, 0}^{F}(k) & \xi_{j, 1}^{F}(k) \cdots & \xi_{j, \varepsilon_{j}-1}^{F}(k)
\end{array}\right] \\
C_{j}^{F}: & =\left[\begin{array}{llll}
x_{j, 0} & x_{j, 1} & \cdots & x_{j, \varepsilon_{j}-1}
\end{array}\right] \\
J_{j}^{F}: & =\left[\begin{array}{ccccc}
0 & 1 & \cdots & 0 & 0 \\
0 & 0 & \cdots & 0 & 0 \\
\vdots & \vdots & \ddots & \vdots & \vdots \\
0 & 0 & \cdots & 0 & 1 \\
0 & 0 & \cdots & 0 & 0
\end{array}\right] \in R^{\varepsilon_{j} \times \varepsilon_{j}}
\end{aligned}
$$

where $j=r+1, r+2, \ldots, m$ and

$$
\begin{gathered}
\Psi_{F}^{\varepsilon}(k):=\left[\begin{array}{lllll}
\Psi_{r+1}^{F}(k) & \Psi_{r+2}^{F}(k) & \cdots & \Psi_{m}^{F}(k)
\end{array}\right] \\
C_{F}^{\varepsilon}:=\left[\begin{array}{lllll}
C_{r+1}^{F}(k) & C_{r+2}^{F}(k) & \cdots & C_{m}^{F}(k)
\end{array}\right] \\
J_{F}^{\varepsilon}:=\text { blockdiag }\left[\begin{array}{lllll}
J_{r+1}^{F}(k) & J_{r+2}^{F}(k) & \cdots & J_{m}^{F}(k)
\end{array}\right]
\end{gathered}
$$

where $\varepsilon:=\sum_{j=r+1}^{m} \varepsilon_{i}$. Then we have the following

Theorem 4. The columns of the following matrix

$$
\begin{gathered}
\Psi_{F}^{\varepsilon}(k):=C_{F}^{\varepsilon}\left(J_{F}^{\varepsilon}\right)^{k}= \\
=\left[\Psi_{r+1}^{F}(k) \Psi_{r+2}^{F}(k) \cdots \Psi_{m}^{F}(k)\right]
\end{gathered}
$$

constitute a solution subspace $B_{F}^{\varepsilon}$ of $B_{D}$ i.e. $B_{F}^{\varepsilon} \subseteq$ $B_{D}-X$ with dimension

$\operatorname{dim} B_{F}^{\varepsilon}=\varepsilon:=$ total sum of the right minimal indices

Proof. Similar to the proof of Theorem 2.

Consider the dual minimal polynomial base of (7). It is easily seen that constitutes a minimal bases of the right null space of the dual polynomial matrix $\tilde{A}(\sigma)$ of $A(\sigma)$, let

$$
\left[\begin{array}{llll}
\tilde{u}_{r+1}(\sigma) & \tilde{u}_{r+2}(\sigma) & \cdots & \tilde{u}_{m}(\sigma)
\end{array}\right]
$$

The greatest degrees of the columns $\tilde{u}_{i}(\sigma), i=r+$ $1, r+2, \ldots, m$ are the same as the right minimal indices of $A(\sigma)$ i.e. $\left\{\varepsilon_{r+1}, \varepsilon_{r+2}, \ldots, \varepsilon_{m}\right\} .{ }^{3}$ Let also

$$
\begin{aligned}
\Psi_{j}^{B}(k): & =\left[\begin{array}{llll}
\xi_{j, 0}^{B}(k) & \xi_{j, 1}^{B}(k) \cdots & \xi_{j, \varepsilon_{j}-1}^{B}(k)
\end{array}\right] \\
C_{j}^{B}:= & {\left[\begin{array}{llll}
x_{j, 0} & x_{j, 1} & \cdots & x_{j, \varepsilon_{j}-1}
\end{array}\right] } \\
J_{j}^{B}:= & {\left[\begin{array}{ccccc}
0 & 1 & \cdots & 0 & 0 \\
0 & 0 & \cdots & 0 & 0 \\
\vdots & \vdots & \ddots & \vdots & \vdots \\
0 & 0 & \cdots & 0 & 1 \\
0 & 0 & \cdots & 0 & 0
\end{array}\right] \in R^{\varepsilon_{j} \times \varepsilon_{j}} }
\end{aligned}
$$

where $j=r+1, r+2, \ldots, m$ and

$$
\begin{gathered}
\Psi_{B}^{\varepsilon}(k):=\left[\begin{array}{llll}
\Psi_{r+1}^{B}(k) & \Psi_{r+2}^{B}(k) & \cdots & \Psi_{m}^{B}(k)
\end{array}\right] \\
C_{B}^{\varepsilon}:=\left[\begin{array}{lllll}
C_{r+1}^{B}(k) & C_{r+2}^{B}(k) & \cdots & C_{m}^{B}(k)
\end{array}\right] \\
J_{B}^{\varepsilon}:=\text { blockdiag }\left[\begin{array}{lllll}
J_{r+1}^{B}(k) & J_{r+2}^{B}(k) & \cdots & J_{m}^{B}(k)
\end{array}\right]
\end{gathered}
$$

Then we have the following

Theorem 5. The columns of the following matrix

$$
\begin{gathered}
\Psi_{B}^{\varepsilon}(k):=C_{B}^{\varepsilon}\left(J_{B}^{\varepsilon}\right)^{N-k}= \\
=\left[\Psi_{r+1}^{B}(k) \Psi_{r+2}^{B}(k) \cdots \Psi_{m}^{B}(k)\right]
\end{gathered}
$$

constitute a solution subspace $B_{B}^{\varepsilon}$ of $B$ i.e. $B_{B}^{\varepsilon} \subseteq$ $B_{D}-X$ with dimension

$\operatorname{dim} B_{B}^{\varepsilon}=\varepsilon:=$ total sum of the right minimal indices

Proof. Similar to the proof of Theorem 3.

Therefore, we conclude that the right minimal indices give rise to $\varepsilon$ linearly independent forward solutions and $\varepsilon$ linearly independent backward solutions which do not belong to $X$.

\subsection{Construction of the whole solution space}

Define the following set of solutions

$x_{j, i}:=\frac{1}{i !} \tilde{u}_{j}^{(i)}(0) \quad i=0,1, . ., \varepsilon_{i}-1$ and $j=r+1, r+2, \ldots, m$

$$
\begin{array}{ll}
\tilde{B}_{F}^{D}=B_{F}^{D} \oplus X & ; \quad \tilde{B}_{B}^{D}=B_{B}^{D} \oplus X \\
\tilde{B}_{F}^{\varepsilon}=B_{F}^{\varepsilon} \oplus X & ; \quad \tilde{B}_{B}^{\varepsilon}=B_{B}^{\varepsilon} \oplus X
\end{array}
$$

Define the vector valued functions

$$
\begin{gathered}
\xi_{j, i}^{B}(k):=\delta(N-k) x_{j, i}+\cdots+\delta(N-(k-i)) x_{j, 0} \\
i=0,1, . ., \varepsilon_{j}-1 \text { and } j=r+1, r+2, \ldots, m
\end{gathered}
$$

where $B_{F}^{D}(k), B_{B}^{D}(k), B_{F}^{\varepsilon}(k)$ and $B_{B}^{\varepsilon}(k)$ are the solution vector spaces which are due to the finite and infinite elementary divisors and the right kernel of $A(\sigma)$. Let also the following spaces

$$
\begin{aligned}
& \hat{B}_{F}^{D}:=\left\{[\xi(k)]_{R}: \xi(k) \in \tilde{B}_{F}^{D}\right\}=\tilde{B}_{F}^{D} / R \\
& \hat{B}_{F}^{\varepsilon}:=\left\{[\xi(k)]_{R}: \xi(k) \in \tilde{B}_{F}^{\varepsilon}\right\}=\tilde{B}_{F}^{D} / R \\
& \hat{B}_{B}^{D}:=\left\{[\xi(k)]_{R}: \xi(k) \in \tilde{B}_{B}^{D}\right\}=\tilde{B}_{B}^{D} / R \\
& \hat{B}_{B}^{\varepsilon}:=\left\{[\xi(k)]_{R}: \xi(k) \in \tilde{B}_{B}^{\varepsilon}\right\}=\tilde{B}_{B}^{\varepsilon} / R
\end{aligned}
$$

3 The vectors $u_{i}(s) i=r+1, . ., m$ are linearly independent and thus its values at $s=0$ i.e. $u_{i}(0) i=r+1, \ldots, m$, are also linearly independent. Therefore the leading coefficient matrix of the vectors $\tilde{u}_{i}(s)$ of the dual polynomial basis are not zero and have the same degrees as the ones of the right minimal polynomial basis of $A(\sigma)$. 
It is obvious that the above spaces partition the sets $\tilde{B}_{F}^{D}, \tilde{B}_{F}^{D}, \tilde{B}_{B}^{D}$ and $\tilde{B}_{B}^{\varepsilon}$ and are vector spaces. Let also

$$
\begin{aligned}
C_{F}:=\left[\begin{array}{ll}
C_{F}^{D} & C_{F}^{\varepsilon}
\end{array}\right] \quad ; \quad J_{F}:=\left[\begin{array}{cc}
J_{F}^{D} & 0 \\
0 & J_{F}^{\varepsilon}
\end{array}\right] \\
C_{B}:=\left[\begin{array}{ll}
C_{B}^{D} & C_{B}^{\varepsilon}
\end{array}\right] \quad ; \quad J_{B}:=\left[\begin{array}{cc}
J_{B}^{D} & 0 \\
0 & J_{B}^{\varepsilon}
\end{array}\right]
\end{aligned}
$$

Theorem 6 . The vector space

$$
\hat{B}_{D}:=B_{D} / R=\hat{B}_{F}^{D} \oplus \hat{B}_{F}^{D} \oplus \hat{B}_{B}^{D} \oplus \hat{B}_{B}^{\varepsilon}
$$

Proof. It is known (1) that the dimension of $\hat{B}_{D}$ is equal to $n+\mu+2 \varepsilon$. In order to determine a basis for $\hat{B}_{D}$ we have developed in the previous three sections a set of $n+\mu+2 \epsilon$ solution vectors that belong to the space $B_{D}-X$ let $\xi_{i}(t)$. Due to the above construction we can show (following similar lines to Chapter 6 of (Karampetakis, 1993)) that these vectors are linearly independent. The proof is based on the fact that the proposed solution vectors are constructed from the linearly independent columns of the unimodular matrix $U_{R}(s)$ and thus the values of $U_{R}(s)$ at $s=0$ are linearly independent. However, in order to avoid all these technicalities we leave the proof. Therefore the vectors $\left[\xi_{i}(k)\right]_{R}$ span the vector space $\hat{B}_{D}$ which verifies the proof.

An interesting conclusion of the above is that

$$
B_{D}=\left\{\begin{array}{c}
\xi(k):=\left[\begin{array}{cc}
C_{F} & C_{B}
\end{array}\right]\left[\begin{array}{cc}
J_{F}^{k} & 0 \\
0 & J_{B}^{N-k}
\end{array}\right]\left[\begin{array}{l}
a \\
b
\end{array}\right]+ \\
+x(k) \\
x(k):=\sum_{i=0}^{k} \hat{u}_{r+1}(i) z_{1}(k-i)+ \\
+\cdots+\sum_{i=0}^{k} \hat{u}_{m}(i) z_{m-r}(k-i) \\
a \in R^{n+\varepsilon}, b \in R^{\mu+\varepsilon}
\end{array}\right\}
$$

\section{CONCLUSIONS}

In this paper we have studied the solution vector space of discrete time nonregular ARrepresentations in an open interval $[0, N]$ and thus extending the results presented in (Gogberg et al., 1982), (Antoniou et al., 1998). More specifically we have shown that the solution space of nonregular AR-representations is divided into equivalence classes where each equivalence class represents the whole number of solutions of the AR-representation under certain boundary values (initial-final conditions). In the sequel we have presented a method for the construction of the behavior space from the structural invariants of the polynomial matrix which describe the system. More specifically a) we have constructed $n+\epsilon$ linearly independent forward solutions due to the finite elementary divisors and the right minimal indices of $A(\sigma)$ and $\mu+\epsilon$ linearly independent backward solutions due to the infinite elementary divisors and the right minimal indices of $A(\sigma)$ and $\mathrm{b}$ ) we have proposed a closed formula for all the solutions of the AR-representation. The meaning of the algebraic structure of a polynomial matrix in relation to the solution vector space of nonregular discrete time AR-representations has thus been elucidated.

The investigation of the solution vector space of discrete nonregular AR-Representations gives rise to a numerous applications as, for example, the solution of the zeroing -output problem, the determination of the controllable or uncontrollable and observable or unobservable subspaces of discrete polynomial matrix descriptions e.t.c. Moreover, we can show following the same reasoning as (Karampetakis and Vardulakis, 1993), (Karampetakis, 1993) that the left kernel of the polynomial matrix which describes the system plays crucial role in the existence of solution.

\section{REFERENCES}

Antoniou, E.N., A.I.G. Vardulakis and N.P. Karampetakis (1998). A spectral characterization of the behavior of discrete time ARrepresentations over a finite time interval.. Kybernetika 34(5), 555-564.

Beelen, T.G.J. (1987). New Algorithms for Computing the Kronecker Structure of a Pencil with Applications to Systems and Control Theory.. PhD thesis. Technische Universiteit Eindhven.

Freeman, H. (1965). Discrete Time Systems : An Introduction to the Theory.. Wiley.

Gogberg, I., P. Lancaster and L. Rodman (1982). Matrix Polynomials. Academic Press.

Karampetakis, N.P. (1993). Notions of Equivalence for Linear Time Invariant Multivariable Systems.. PhD thesis. Department of Mathematics, Aristotle University of Thessaloniki, Greece.

Karampetakis, N.P. (2001). On the determination of the dimension of the solution space of discrete time AR-representations.. submitted for possible presentation to the 15th IFAC World Congress.

Karampetakis, N.P. and A.I.G. Vardulakis (1993). On the behaviour of continuous-time ARrepresentations.. Second European Control Conference, Groningen, The Netherlands. pp. 1784-1789. 